\title{
Traumatic rupture of solid pseudopapillary tumors of the pancreas in children: A case report
}

\author{
SEMIH LÜTFI MIRAPOĞLU ${ }^{1}$, IBRAHIM AYDOGDU ${ }^{1}$, ZUHAL GUCIN $^{2}$, \\ TEMEL FATIH YILMAZ ${ }^{3}$, TARIK UMUTOGLU ${ }^{4}$ and HUSEYIN KILINCASLAN ${ }^{1}$
}

Departments of ${ }^{1}$ Pediatric Surgery, ${ }^{2}$ Pathology, ${ }^{3}$ Radiology and ${ }^{4}$ Anesthesiology and Reanimation, Bezmialem Vakif University Faculty of Medicine, Istanbul 34093, Turkey

Received February 29, 2016; Accepted June 13, 2016

DOI: $10.3892 / \mathrm{mco} .2016 .997$

\begin{abstract}
Solid pseudopapillary tumor (SPT) of the pancreas is a pathological entity rarely encountered in children. Despite its malignant characteristics, SPT has a favorable clinical prognosis. This tumor is more frequently observed in females and is commonly localized in the tail and body of the pancreas. This is the case report of a 9-year-old female patient who presented with severe abdominal pain of sudden onset and vomiting following blunt abdominal trauma. Upon physical examination, abdominal distension, tenderness and abdominal guarding were detected. Abdominal magnetic resonance imaging revealed a mass sized $8 \times 9 \times 12 \mathrm{~cm}$ in the pancreas, with hemorrhagic-necrotic areas, and abundant intra-abdominal fluid. Intra-abdominal exploration confirmed the presence of an abundant amount of intraperitoneal hemorrhagic fluid. A mass was identified, originating from the body and the tail of the pancreas, adherent to the mesentery of the colon and the hilum of the spleen, which had ruptured in its inferolateral side. The tumor was extirpated together with the spleen and the involved parts of the pancreas. Histopathological examination demonstrated a solid pseudopapillary tumor of pancreatic origin. The patient's postoperative course was uneventful, clinically as well as metabolically.
\end{abstract}

\section{Introduction}

Solid pseudopapillary tumors (SPTs) of the pancreas are a rare occurrence, accounting for $1-2 \%$ of all solid pancreatic tumors (1). SPT is more prevalent in females and is more frequently encountered in adolescence. The clinical findings of SPT vary considerably (2). The patients are often diagnosed during work-up for non-specific signs and symptoms, such as

Correspondence to: Dr Semih Lütfi Mirapoğlu, Department of Pediatric Surgery, Bezmialem Vakif University Faculty of Medicine, Adnan Menderes Boulevard, Fatih, Istanbul 34093, Turkey

E-mail: semihmirap@gmail.com

Key words: pseudopapillary tumor, pancreas, trauma, acute abdomen intra-abdominal mass, abdominal pain, and/or discomfort. The patients rarely present with an acute abdomen resulting from the rupture of the tumor capsule. In our patient, the tumor had ruptured as a result of blunt abdominal trauma. The patient underwent laparotomy with the indication of an acute abdomen, during which an SPT was identified and completely resected.

\section{Case report}

A 9-year-old female patient presented to the emergency service with severe abdominal pain of sudden onset and vomiting following blunt abdominal trauma (impact of a swing on her abdomen). Upon physical examination, the patient was found to have abdominal distension, tenderness, abdominal guarding and elevated body temperature $\left(>37.9^{\circ} \mathrm{C}\right)$; she was also tachycardic and mildly hypotensive (blood pressure $80 / 55 \mathrm{mmHg}$ ). The laboratory tests results revealed anemia $(\mathrm{Hb} 7.9 \mathrm{mg} / \mathrm{dl})$ and leukocytosis (white blood cell count $22.800 / \mathrm{mm}^{3}$ ). An abdominal X-ray revealed dilated bowel loops and air-fluid levels. On abdominal ultrasound, a mass measuring 120x90 mm was identified on the pancreas, together with a generalized intra-abdominal fluid collection. Abdominal magnetic resonance imaging revealed a mass sized 8x9x12 $\mathrm{cm}$ located in the pancreas, with hemorrhagic-necrotic areas and abundant intra-abdominal fluid. The mass appeared to originate from the inferolateral aspect of the pancreas (Fig. 1). The patient underwent surgery following resuscitation. Intra-abdominal exploration revealed an abundant amount of intraperitoneal hemorrhagic fluid. A mass originating from the body and tail of the pancreas was identified, which was adherent to the mesentery of the colon and the hilum of the spleen, and had ruptured from its inferolateral side. The mass was dissected from the colonic mesentery but could not be separated from the splenic vessels. Therefore, the tumor was extirpated together with the spleen and the involved parts of the pancreas (Fig. 2). Approximately $40 \%$ of intact, healthy pancreatic tissue remained. Histopathological examination demonstrated a solid pseudopapillary tumor of pancreatic origin (Fig. 3). The patient's postoperative course was clinically and metabolically uneventful. Written informed consent was obtained from the patient's parents for the publication of this case report and related images. 


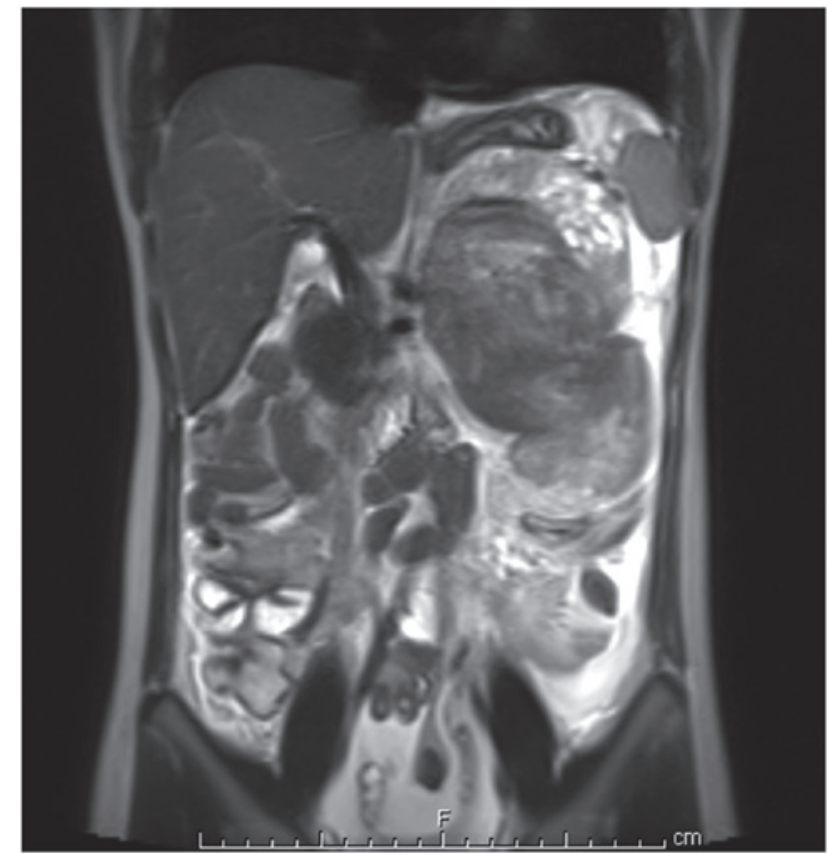

Figure 1. An abdominal magnetic resonance imaging demonstrated a mass sized $8 \times 9 \times 12 \mathrm{~cm}$ in the pancreas (arrow), with hemorrhagic-necrotic areas and abundant intra-abdominal fluid. The mass appeared to originate from the inferolateral aspect of the pancreas.

\section{Discussion}

Although SPT is usually located in the tail and body of the pancreas (3-5), the caput pancreas is also a frequent location (6). Patients with SPT may present with complaints of abdominal pain and discomfort, and the diagnosis of SPT may be incidental $(3,6)$. In addition, a proportion of patients are incidentally diagnosed with SPT during work-up for hemorrhagic complications and peritonitis following abdominal trauma $(5,7,8)$. However, spontaneous perforation of the tumor without a history of trauma has been reported (9). An increased use of radiological modalities has resulted in a 7 -fold increase in the number of patients diagnosed with SPT since 2000 (3).

According to the World Health Organization classification, SPT is considered to be a low-grade exocrine pancreatic malignancy $(1,10)$. Hu et al reported that gender, preoperative age of the patient and tumor size are not associated with the malignant potential of SPT (11). However, in a recent study, the authors stated that a tumor with a diameter of $>5 \mathrm{~cm}$ as visualized on computerized tomography (CT) scan may be an indication of malignancy (10). However, in another study, the solid component of the tumor was considered to be a significant predictor of malignant potential (4). Hwang et al emphasized that a SPT may be classified as benign or malignant based on various criteria, including the presence/extent of perineural invasion, angioinvasion, capsular invasion, lymph node involvement, adjacent organ invasion and distant metastases (4). Rupture and metastasis of the tumor are the primary causes of recurrence. Vascular involvement has also been reported in cases with SPT, and the tumor most commonly metastasizes to the peritoneum, liver and lymph nodes $(3,4,10)$.

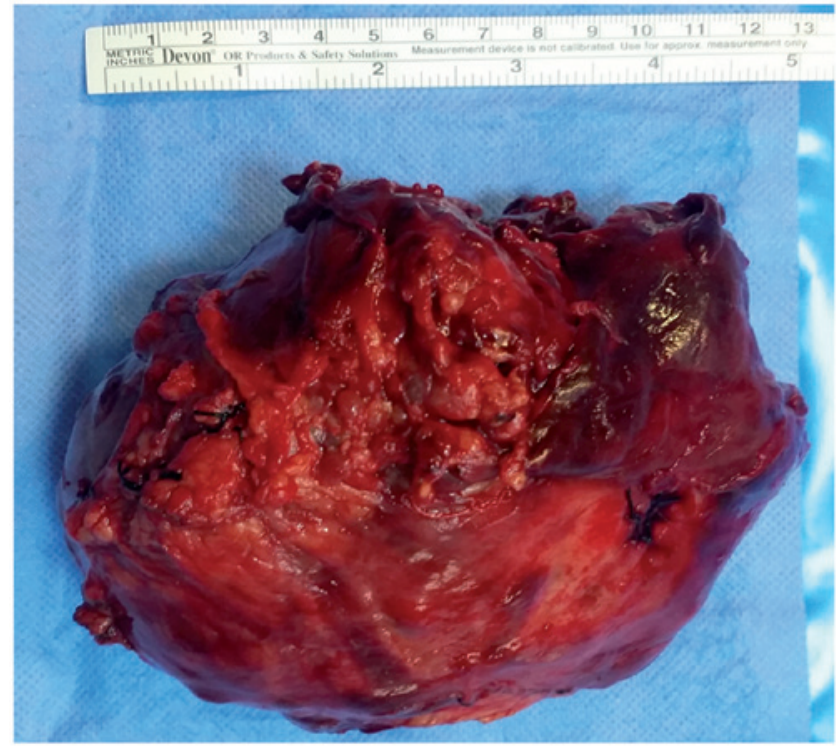

Figure 2. Macroscopic appearance of the resected tumor.

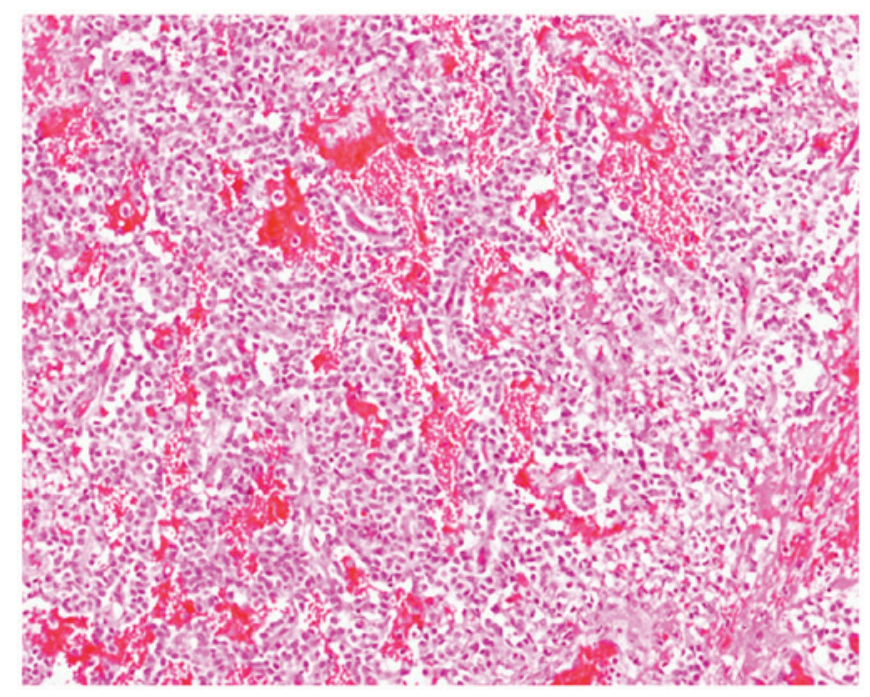

Figure 3. Histopathological findings of the tumor. Pseudopapillary projections covered with one or more layers of cells were observed, with extensive hemorrhagic areas.

The treatment of SPT consists of total surgical excision of the tumor mass and the adjacent pancreatic tissue. Enucleation is not recommended, as it carries the risk of incomplete resection. A statistically significant difference between laparoscopic and open surgery was not detected with regard to postoperative complications and prognosis $(2,11,12)$. Local recurrence often occurs when the tumor is incompletely resected or ruptures (4). In recurrent or metastatic cases, chemotherapy and/or radiotherapy may be effective, whereas 5-fluorouracil and gemcitabine are used as adjuvant chemotherapy (3). Since very few cases require radiotherapy and/or chemotherapy, it is difficult to evaluate the outcomes of these treatments (5). Hwang et al reported on their cases of liver transplantation for patients with multiple liver metastases (4). The average patient follow-up period was reported to be $36-67$ months $(3,6,10)$ whereas in 1 patient the follow-up period was 7 years (5). 
Among pediatric patients, SPT rarely presents with traumatic rupture. Tajima et al (7) presented the case of a 12-year-old female patient who presented with an acute abdomen secondary to abdominal trauma. SPT rupture was detected in the patient, and she underwent urgent surgery for hemostasis, drainage and biopsy. The biopsy result was consistent with SPT. Five weeks later, the patient underwent an exploratory laparotomy, and the mass was found to be localized to the head of the pancreas; it had not metastasized into the intra-abdominal cavity, and the surrounding organs were totally extirpated with pylorus-sparing pancreoduodenectomy. At the 7-year postoperative follow-up visit, an abdominal CT scan revealed metastatic nodules; these were subsequently removed with a third operation and pathologically confirmed to be SPT recurrence.

Park et al (13) reported the case of another pediatric patient with hemoperitoneum due to SPT rupture. During the first operation, laparotomy was performed that revealed the presence of an unresectable mass. Therefore, only hemostatic control and a biopsy were performed. Subsequently, the patient received 3 cycles of chemotherapy. Three months later, during a second-look laparotomy, the patient underwent subtotal pancreatectomy and splenectomy. During the postoperative follow-up visit, portal vein thrombosis and liver metastasis were detected, necessitating additional chemotherapy with radiofrequency ablation. On a follow-up visit 97 months later, the patient was disease-free, although she exhibited portal vein obliteration and cavernous transformation.

Our patient presented with similar manifestations. A one-stage operation was performed, during which the patient received subtotal pancreactomy, splenectomy and hemostatic control. No additional treatment was applied. The 1-year follow-up visit revealed no medical problems. This patient remains under close surveillance.

In conclusion, pancreatic SPT is a rare tumor with a low malignant potential. The optimal treatment for the tumor is total surgical excision. Patients with metastatic disease or a ruptured SPT should be closely followed up for tumor recurrence. Tumor markers have not been found to be helpful for postoperative monitorıng (14).

\section{References}

1. Bhatnagar R, Olson MT, Fishman EK, Hruban RH, Lennon AM and Ali SZ: Solid-pseudopapillary neoplasm of the pancreas: Cytomorphologic findings and literature review. Acta Cytol 58: 347-355, 2014.

2. Takamatsu S, Nagano H, Ohtsukasa S, Kawachi Y and Maruyama H: A case of spontaneous ruptured solid pseudopapillary tumor of pancreas resected by laparoscopic surgery. Case Rep Med 2013: 953240, 2013.

3. Law JK, Ahmed A, Singh VK, Akshintala VS, Olson MT, Raman SP, Ali SZ, Fishman EK, Kamel I, Canto MI, et al: A systematic review of solid-pseudopapillary neoplasms: Are these rare lesions? Pancreas 43: 331-337, 2014.

4. Hwang J, Kim DY, Kim SC, Namgoong JM and Hong SM: Solid-pseudopapillary neoplasm of the pancreas in children: Can we predict malignancy? J Pediatr Surg 49: 1730-1733, 2014.

5. Huang HL, Shih SC, Chang WH, Wang TE, Chen MJ and Chan YJ: Solid-pseudopapillary tumor of the pancreas: Clinical experience and literature review. World J Gastroenterol 11: 1403-1409, 2005.

6. Suzuki S, Hatori T, Furukawa T, Shiratori K and Yamamoto M: Clinical and pathological features of solid pseudopapillary neoplasms of the pancreas at a single institution. Dig Surg 31: 143-150, 2014.

7. Tajima Y, Kohara N, Maeda J, Inoue K, Kitasato A, Natsuda K, Irie J, Adachi T, Kuroki T, Egochi S and Kanematsu T: Peritoneal and nodal recurrence 7 years after excision of a ruptured solid pseudopapillary neoplasm of the pancreas: Report of a case. Surg Today 42: 776-780, 2012.

8. Spătaru RI, Enculescu A and Popoiu MC: Gruber-Frantz tumor: A very rare pathological condition in children. Rom J Morphol Embryol 55: 1497-1501, 2014.

9. Pattanshetti VM, Vinchurkar K and Pattanshetti SV: Solid pseudo papillary tumor of pancreas: Presenting as acute abdomen in a female child. Indian J Med Paediatr Oncol 35: 184-186, 2014.

10. Kim MJ, Choi DW, Choi SH, Heo JS and Sung JY: Surgical treatment of solid pseudopapillary neoplasms of the pancreas and risk factors for malignancy. Br J Surg 101: 1266-1271, 2014.

11. Hu S, Lin X, Song Q and Chen K: Solid pseudopapillary tumour of the pancreas in children: Clinical and computed tomography manifestation. Radiol Med 117: 1242-1249, 2012.

12. Morita K, Urushihara N, Fukumoto K, Miyano G, Yamoto M, Nouso H, Miyake H and Kaneshiro M: Solid pseudopapillary tumor of the pancreas in children: Surgical intervention strategies based on pathological findings. Pediatr Surg Int 30: 253-257, 2014.

13. Park JY, Kim SG and Park J: Solid pseudopapillary tumor of the pancreas in children: 15-year experience at a single institution with assays using an immunohistochemical panel. Ann Surg Treat Res 86: 130-135, 2014.

14. Yagcı A, Yakan S, Coskun A, Erkan N, Yıldırım M, Yalcın E and Postac1 H: Diagnosis and treatment of solid pseudopapillary tumor of the pancreas: Experience of one single institution from Turkey. World J Surg Oncol 11: 308, 2013. 